\title{
DYNAMISCHE ENERgIEMODELLE \\ als Planungs- und Entscheidungshilfe \\ DARGESTELLT AN EINEM ENERGIEMODELL FÜR DIE BRD*)
}

\author{
A. Voss \\ St. Rath-Nagel \\ K. Schmitz \\ G. Kolb \\ D. Elsinghorst \\ G. Egberts \\ Programmgruppe Systemforschung und Technologische Entwicklung \\ der Kernforschungsanlage Jülich G.m.b.H.
}

Die Entwicklung und Anwendung mathematischer Modelle zur Analyse komplexer Probleme ist der breiten రffentlichkeit im wesentlichen durch die vom club of Rome initiierten Arbeiten $\mathrm{zu}$ den weltweiten Wachstumsproblemen bekannt geworden. Dennoch herrscht gerade hier und auch bei den Gruppen, die im Rahmen ihrer Entscheidungsfindung Modelle und insbesondere Energiemodelle anwenden oder einsetzen sollen, wenig Klarheit über deren Aussagemöglichkeiten und verbunden damit über deren Gestalt und Struktur.

Damit das nachfolgend vorgestellte Energiemodell, die mit ihm gewonnenen Ergebnisse und die Anwenđungsmöglichkeiten richtig verstanden werden, sollen daher zu Anfang dieser Ausführungen die Motivation für die Entwicklung von Energiemodellen, die Einschätzung der zu erzielenden Ergebnisse und insbesondere die Zielsetzungen erläutert werden.

Die Probleme der Energieversorgung, mit denen die Welt heute konfrontiert ist, lassen sich durch Schlüsselbegriffe wie

- Wachstum des Energieverbrauchs,

- zunehmende Abhängigkeit der Wirtschaft und folglich des materiellen Lebensstandards des einzelnen von einer gesicherten Energiedarbietung

- Erschöpfung der fossilen Energiereserven

- zunehmende Importabhängigkeit und in Zusammenhang damit Fragen der Versorgungssicherheit

*) Diese Arbeit wird finanziell von der Stiftung Volkswagenwerk unterstūtzt. 

systeme

kennzeichnen.

Diese Aufzählung allein macht schon deutlich, daB die Energieversorgung nicht länger als ein isoliertes, technisch-ökonomisches Problem angesehen werden kann. Eine systemorientierte $\mathrm{Be}-$ trachtungsweise scheint erforderlich, die ausgeht von der Energieversorgung als ein in eine technisch-ökonomisch-ökologischgesellschaftliche Umgebung eingebettetes System, dessen Entwicklung wesentlich von seinen Wechselwirkungen mit eben dieser Umgebung bestimmt wird. Für die Beurteilung und Planung der zukünftigen alternativen Energieversorgungssysteme ist also die Berücksichtigung der Einbettung des Energiesystems in seine Umgebung die generelle Forderung, die sich aus einer solchen systemorientierten problembetrachtung ableitet.

Die Entwicklung mathematischer Modelle scheint ein besonders geeignetes Hilfsmittel für die Erarbeitung von derartigen Entscheidungshilfen zu sein, und zwar von Entscheidungshilfen, die im Prinzip der Vielzahl der zu berücksichtigenden Zusammenhänge und der inneren Verkettung der Probleme gerechter werden als andere z.B. intuitive oder isolierte disziplinäre Analysemethoden. Formal bringen mathematische Modelle zwei Vorteile mit sich:

- Erstens zwingt die Notwendigkeit der mathematischen Formulierung der Zusammenhänge und der Systemstruktur zu einer intensiven Beschäftigung mit den Problemen, was in der Regel ein besseres Problemsverständnis zur Folge hat,

- und zweitens bieten mathematische Modelle bessere Voraussetzungen, die zugrundeliegenden Hypothesen und die verwendeten zusammenhänge zu diskutieren und die Auswirkungen veränderter Parameter aufzeigen zu können.

Ein Modell ist dabei notwendigerweise immer ein vereinfachtes Abbild des realen Systems, denn eine exakte Abbildung des Systems in einem Modell würde bedeuten, daß ein Modell den gleichen Komplexitätsgrad hätte wie das reale System; es wäre damit ebenso wenig operationalisierbar wie das system selbst. Hieraus leitet sich die Forderung $\mathrm{ab}$, daB ein Modell nur die relevanten Elemente und ihre wechselseitigen Beziehungen enthalten muB. Welches sind aber nun die relevanten Elemente und Beziehungen? Diese Frage ist nur im Zusammenhang mit der Zielsetzung des Modells, d.h. den Frage- und Problemstellungen, die mit dem Modell analysiert werden sollen, beantwortbar. Die Definition der ziele einer Modellentwicklung ist deshalb der erste notwendige Schritt, der vor allen anderen Arbeiten zu erfolgen hat, denn die Zielsetzungen sind für die Modellelemente und -struktur sowie für den Aggregationsgrad der Modellgrößen bestimmend. Ebenso muB sich die Auswahl des methodischen Ansatzes wie zum Beispiel der linearen oder dynamischen Optimierung, der Input-Output-Rechnung oder der Simulation nach den Zielen der Modelluntersuchung richten. Es gibt deshalb schlechthin keine "beste" Methode. Ein anderer Punkt, der für die Modellentwicklung wich- 
tig erscheint, ist die Integration der Modellentwicklungsarbeiten in andere Arbeiten und Untersuchungen. Erst eine enge Wechselwirkung von Einzelstudien, die Daten und Resultate für das Modell bereitstellen, mit der Modellentwicklung selbst kann zu gesicherten und qualitativ guten Ergebnissen führen.

Bevor nun die Modelle, die im Rahmen der Arbeiten der Programmgruppe Systemforschung und Technologische Entwicklung der Kernforschungsanlage Jülich entwickelt worden sind, năher vorgestellt werden, soll daher zunächst stichpunktartig auf die übergeordneten zielsetzungen eingegangen werden.

Das übergeordnete Ziel der Modellentwicklungsarbeiten ist es, ein Instrumentarium für die Analyse der langfristigen Entwicklungsmöglichkeiten des Energiesystems der BRD zu schaffen. Dabei sollen insbesondere die Wechselwirkungen des Systems mit seiner Umgebung, d.h. mit der gesamten Wirtschaft, der Gesellschaft und der Umwelt berücksichtigt werden. Dieses allgemeine ziel läBt sich in mehrere Teilziele aufgliedern, wie Bild 1 zeigt:

1. Untersuchung der Struktureigenschaften und des dynamischen Verhaltens im Bereich Energienachfrage und Energieangebot.

2. Erforschung und Aufzeigen von Grenzsituationen und kritischen Zuständen in der Energiebedarfsdeckung, wie z.B. Untersuchung der Auswirkungen einer Versorgungskrise.

3. Aufzeigen der Folgen und Bewertung alternativer Energieversorgungsstrategien.

4. Untersuchung von Eingriffs- und Steuerungsmöglichkeiten in der Entwicklung der Energiewirtschaft wie z.B. Veränderung des wirtschaftlichen Wachstums oder Einführung von Energieeinsparmaßnahmen.

Diese ziele sollen mit einem Modell erreicht werden, das in seiner Endphase die in Bild 2 angegebenen Modellsektoren enthalten soll.

Der Wirtschaftssektor beschreibt dynamisch die Entwicklung einzelner Wirtschaftsbereiche. Ausgangspunkt ist dabei die Endnachfrage und die tber eine intersektorale Vorleistungsverflechtung ermittelte $\mathrm{W}$ wischennachfrage in den einzelnen wirtschaftsbereichen. Die Gesamtnachfrage bildet die Grundlage für die Vergrösserung des Produktionskapitals und schafft damit eine der notwendigen Bedingungen für die Erhöhung der Nettoproduktion, deren Summe uber alle wirtschaftsbereiche (korrigiert um den saldo der Erwerbs- und Vermögenseinkommen zwischen Inländern und der übrigen Welt) das Bruttoinlandsprodukt bildet.

Im Bevölkerungssektor wird die Entwicklung der Bevölkerung und die als Arbeitskrăfteangebot zur Verfügung stehende Zahl der Erwerbspersonen ermittelt.

Ausgehend von den in diesen beiden Sektoren ermittelten Grunddaten, wie z.B. der Nettoproduktion der einzelnen wirtschafts- 
zweige, des verfügbaren Einkommens pro Kopf der Bevölkerung, der Zahl der Wohnungen und der Verkehrsleistung pro Kopf bzw. pro Industrieproduktionseinheit werden im Energiebedarfssektor der sektorale Energiebedarf der Industrie sowie der Energiebedarf im Verkehr und in den Sektoren Haushalte und Kleinverbrauch quantifiziert.

Im Substitutionssektor erfolgt die Aufteilung des Energiebedarfs auf die einzelnen Endenergieträger und die Darstellung der Substitutionsvorgänge auf dem Energiemarkt.

Aufgabe des Finanzierungs- und Investitionssektors ist es, die Investitionsvorhaben und den Finanzierungsbedarf der Energiewirtschaft in den gesamtwirtschaftlichen Bedarf an Finanzmitteln einzubetten, um eventuell finanzielle Grenzen des Ausbaus der Energieversorgung aufzuzeigen.

Ziel des Energieversorgungssektors ist die Ermittlung optimaler Ausbaustrategien des Energieversorgungssystems unter Berücksichtigung der jahres- und tageszeitlichen Schwankungen des Energiebedarfs und der stochastischen bzw. periodisch wechselnden Verfügbarkeit einiger Primärenergieträger (z.B. Wasserkraft, Sonnenenergie).

Der Umweltsektor dient der Quantifizierung der Umweltsituation sowie der Beschreibung der Rückwirkungen der Umweltbelastungen auf den Energieverbrauch und die Energieverbrauchsstruktur.

Die Berücksichtigung weltweiter, für die Energiewirtschaft der BRD dominanter Entwicklungen - z.B. der Preis- und Versorgungssituation nicht heimischer Primärenergieträger oder globaler ökologischer Veränderungen - erfolgt mit Hilfe bereits entwikkelter globaler Energie- und Umweltmodelle, die zur exogenen Festlegung der Import- und Exportströme verwendet werden. Die im Bild dargestellten von außen wirkenden Pfeile sollen dies andeuten.

Damit ist der Endzustand der Modellkonzeption umrissen. Bild 3 zeigt demgegenüber in der derzeitig vorliegenden und abgeschlossenen Fassung die Grundstruktur des Energiemodells der BRD, also die wesentlichen strukturelemente und deren funktionale Verknüpfung.

Aus Gründen der Uberschaubarkeit und der mathematischen Handhabung einzelner Blöcke ist das gesamte Modell in 8 Sektoren strukturiert, wobei diese Sektoreneinteilung in der derzeitigen Fassung nicht mit der Eintellung des vorangegangenen Bil-
des identisch ist.

Will man, wie in den Zielen erläutert, das dynamische Verhalten im Bereich der Energienachfrage darstellen, so ist zunächst nach den Determinanten des wirtschaftlichen Wachstums zu fragen. Für eine detaillierte Erklärung der wirtschaftlichen Entwicklung ist neben der Beschreibung der Wachstumsprozesse auch die Darstellung von Strukturänderungen zwischen den einzelnen Wirtschaftsgruppen erforderlich. Es wurde daher ein Ansatz gewählt, der von einer Disaggregierung der Wirtschaft in sechs Bereiche 
ausgeht. Es sind dies zunächst einmal vier industrielle Bereiche, nämlich die

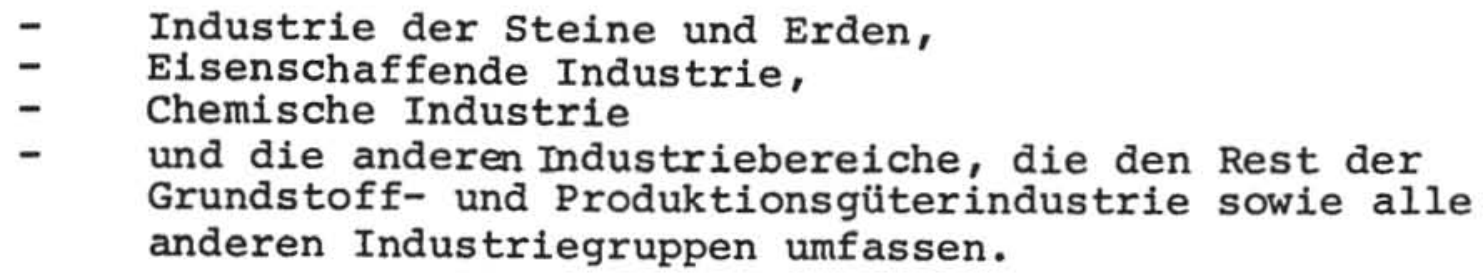

Daruber hinaus existiert der Bereich der Energiewirtschaft, der zusätzlich in sechs Untergruppen unterteilt wurde, und schließlich ein Bereich, zu dem alle anderen Unternehmen gerechnet werden, die weder der Industrie noch der Energiewirtschaft angehören. Dies sind Landwirtschaft, Industriebetriebe mit weniger als 10 Beschäftigten, Baugewerbe, Handel, Verkehr, Nachrichten, Dienstleistungen, Wohnungsvermietung und staatliche Bereiche.

Der Energieverbrauch wird im sechsten Sektor für die eben erwähnten vier Industriegruppen, für den Verkehr (getrennt nach Güter- und Personenverkehr und Verkehrsträgern), für den Bereich der Haushalte (getrennt nach Sammelheizung, Einzelheizung und übrigen Verbrauch) und für die Gruppe Kleinverbraucher festgelegt. Dabei werden beim derzeitigen Ausbauzustand des Modelis 6 verschiedene Primär- und 11 verschiedene Sekundärenergieträger betrachtet. Der Umwelsektor dient dazu, die Emissionen für acht Schadstoffe zu ermitteln. (Die Hintereinanderschachtelung der Symbole der einzelnen Modellparameter soll andeuten, daB die Struktur in den jeweiligen Gruppen für jede Gruppenvariable dieselbe ist.)

Im Bevölkerungs- und Beschäftigungssektor wird ausgehend von der Bevölkerungsentwicklung die $\mathrm{Zahl}$ der Beschäftigten in den produzierenden Bereichen durch einen Vergleich des Arbeitskräfteangebots, das aus der Bevölkerungszahl und vorgegebenen Erwerbsquoten ermittelt wird, mit der Arbeitskräftenachfrage der Unternehmen festgelegt. Die Arbeitskräftenachfrage ist dabei von den Produktionsbedingungen in den einzelnen Bereichen abhängig; sie läBt sich uber die Kapitalintensität, d.h. die pro Kapitaleinheit eingesetzten Arbeitskräfte bei Kenntnis des zugehörigen Kapitalstocks ermitteln.

Im zweiten Sektor wird der Produktionsfaktor Kapital, gerechnet in realen Preisen (Preisbasis 1962), für die einzelnen Wirtschaftsbereiche bestimmt. Der Kapitalstock vermehrt sich dabei um die Bruttoinvestitionen und vermindert sich um die abnutzungsbedingten Abschreibungen, die im Bild aus Vereinfachungsgründen nicht aufgenommen wurden.

Beschäftigte und eingesetztes Produktionskapital sind Produktionsfaktoren, durch deren Einsatz das Produktionsergebnis, gemessen als Beitrag zum Bruttoinlandsprodukt, bestimmt wird. Als dritte den Produktionsoutput bestimmende GröBe wirkt der technische Fortschritt, der aus Gründen einer möglichst geschlossenen Erklärung nicht exogen vorgegeben, sondern über eine Korrelation mit den kumulierten Bruttoinvestitionen bestimmt wird. Der Beitrag zum Bruttoinlandsprodukt bzw. die Nettoproduktion 
der einzelnen Bereiche wird uber eine Produktionsfunktion vom Cobb-Douglas-Typ ermittelt.

Aufgabe des vierten Sektors, des Nachfragesektors, ist die Ermittlung der End- und Zwischennachfrage nach Gütern und Dienstleistungen der einzelnen Wirtschaftsbereiche. Die Endnachfrage besteht aus vier Komponenten, der Nachfrage für

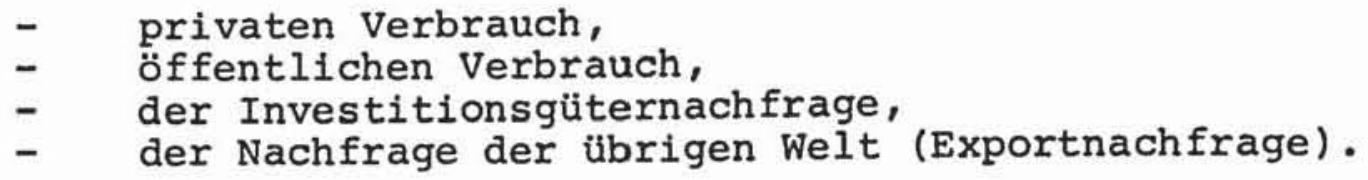

Die einzelnen Komponenten der Endnachfrage werden dabei im Falle des privaten und öffentlichen Verbrauchs indirekt aus dem Bruttoinlandsprodukt und für den Export über vorgegebene Exportquoten ermittelt. Die Investitionsgüternachfrage wird im fünften Sektor determiniert, der nachfolgend näher beschrieben wird. Die für die Befriedigung der Endnachfrage notwendigen Vorleistungen der einzelnen Wirtschaftsbereiche, also die Zwischennachfrage der Produktionsbereiche, wird mit einer auf die sechs Wirtschaftsbereiche aggregierten Input-Output-Matrix errechnet.

Ist die Nachfrage berechnet, so lassen sich die Investitionsentscheidungen der Unternehmen aufgrund einer auf der vergangenen Entwicklung aufbauenden Nachfrageeinschätzung festlegen, wenn man von anderen das Investitionsverhalten beeinflussenden Faktoren absieht. Die Investitionsaufträge werden also durch die für die Deckung einer bestimmten erwarteten Nachfrage erforderlichen Veränderung des Kapitalstocks ermittelt. Die so bestimmten Investitionsaufträge beeinflussen dabei in zweierlei Weise das gesamte Wachstumsverhalten. Einerseits stellen sie selbst einen Teil der Endnachfrage dar, und andererseits vermehren die fertiggestellten Investitionsgüter den Produktionsfaktor Kapital. Dabei schließt sich hier ein wichtiger Regelkreis, der von den Investitionen, über den Kapitalstock, den Beitrag zum BIP und die Nachfrage wieder zu den Investitionsaufträgen führt.

Im sechsten Sektor werden die in der Industrie, in den Haushalten, bei den Kleinverbrauchern und im Verkehr verbrauchten Energiemengen errechnet. Dabei wird für die vier industriellen Bereiche nach dem energetischen und nicht-energetischen Verbrauch unterschieden. Bei den Haushalten wird eine Trennung nach den Verwendungszwecken Raumheizung und übriger Energieverbrauch vorgenommen, und im Verkehr wird der Energieverbrauch den einzelnen Verkehrsträgern der Bereiche Personen- und Güterverkehr zugeord-
net.

Als Bestimmungsgrößen für den industriellen Energieverbrauch werden die jeweiligen Produktionszahlen und die mit den kumulierten Investitionen korrelierten spezifischen Energieverbräuche verwendet. Der Energieverbrauch der Haushalte, der Kleinverbraucher und des Verkehrs wird durch aus der wirtschaftlichen Entwicklung abgeleitete Größen, d.h. durch das verfügbare Einkommen, den Wohnungsbestand und das Verkehrsaufkommen im Personen- und Güterverkehr erklärt. Der so errechnete Endenergieverbrauch wird 
anschlieBend durch Strukturfaktoren auf die angebotenen Endenergieträger aufgeteilt.

Die wesentliche Aufgabe des Energieversorgungssektors besteht in der Bestimmung des für einen bestimmten Verbrauch an Endenergie notwendigen Primärenergieeinsatzes. Dabei wird ein Teil der Endenergie iber die verschiedenen Umwandlungsverfahren aus der Primärenergie gewonnen, z.B. Strom aus Kohle, Erdgas, Heizöl, Wasserkraft oder Kernenergie, und der andere Teil der Endenergie wird von den nicht umgewandelten primärenergieträgern gestellt. Dies ist z.B. der Fall bei der direkten Nutzung des Erdgases zur Raumheizung. AuBerdem ist hierbei noch der Energieverbrauch der Energiewirtschaft, also die Energie, lie in den verschiedenen Stufen der Gewinnung, Umwandlung und Verteilung von Energie selbst verbraucht wird, zu berücksichtigen. Mit Hilfe von vorgegebenen Importanteilen und Produktionsgrenzen für die inländische Produktion läBt sich der gesamte Primärenergiebedarf in die Komponenten Energieimporte und Energiegewinnung im Inland aufteilen. Zusammen mit dem Umwandlungseinsatz und dem Aufwand für die Energievertellung kann somit die Produktion in allen Teilbereichen der Energiewirtschaft (Gewinnung, Umwandlung und Verteilung) angegeben werden. Sie erfordert ebenso wie die Produktion in den anderen Wirtschaftsbereichen den Einsatz von Beschäftigten, von Kapital und die Bereitstellung von Vorleistungen zur Befriedigung der Zwischennachfrage der Energiewirtschaft. Andererseits trägt auch die Energiewirtschaft zur Einkommensschaffung und zum Bruttoinlandsprodukt bei, und ihre Investitionsaufträge sind Teil der Endnachfrage. Hier erfolgt also wieder die Kopplung der Energiewirtschaft, die aus Gründen einer detaillierten energetischen Betrachtung gesondert behandelt wird, an die anderen Wirtschaftsbereiche und an die im Modell dargestellten wirtschaftlichen Entwicklungsmechanismen.

Mit Hilfe der industriellen Produktion und des Energieverbrauchs nach Energieträgern und Verbrauchssektoren werden im Umweltsektor uber spezifische Emissionsfaktoren die Freisetzungsmengen der wichtigsten Schadstoffe bestimmt. Gegenüber Ansätzen, die die UmweltschutzmaBnahmen als Funktion der Zeit vorgeben, ist in diesem Modell versucht worden, die EmissionsreduzierungsmaBnahmen, d.h. die Entwicklung der spezifischen Emissionsfaktoren, unter Einbeziehung gesellschaftlicher und interessenpolitischer Verhaltungsmechanismen zu simulieren. Prinzipiell wird dabei wie folgt vorgegangen. Die verschiedenen Schadstoffemissionen sind die bestimmenden GröBen der Umweltsituation bzw. der Umweltbelastung. Das BewuBtwerden einer nicht tragbaren oder nicht gewinschten Umweltsituation würde, unter der Hypothese der Existenz eines Umweltschadens- und -bewuBtseins-Regelkreises, nach einiger Zeit zur Verordnung von Emissionsreduzierungsmaßnahmen, oder was verglelchbar damit ist, zur Aufstellung von Umweltstandards fuhren, die wiederum die spezifischen Emissionsfaktoren herabsetzen.

Diese Ausführungen sollen zur Erklärung der Grundstruktur dienen; die einzelnen Sektoren des Modells sind strukturell weiter aufgegliedert und enthalten eine Vielzahl von Funktionszusammenhängen, die hier nicht im einzelnen erläutert werden können. FaBt man die vorstehenden Ausfühungen zusammen, so läBt sich der erarbeitete Modellansatz wie folgt kennzeichnen: 
1. Es handelt sich um ein quasi-geschlossenes dynamisches Simulationsmodell aus einem System gekoppelter Differentialgleichungen.

2. Die wirtschaftliche Entwicklung wird für sechs wirtschaftsbereiche durch eine Cobb-Douglas-Produktionsfunktion mit einer endogenen Erklärung für die Produktionsfaktoren Arbeit, Kapital und Technischer Fortschritt beschrieben. Die Investitionen werden aus der Nachfrageentwicklung der Vergangenheit abgeleitet, wobei die Nachfrage auch die uber eine intrasektorale Vorleistungsverflechtung ermittelte Zwischennachfrage enthält.

3. Der Energieverbrauch wird für die einzelnen Verbrauchsgruppen durch aus der wirtschaftlichen Entwicklung abgeleitete Faktoren, d.h. Produktionsziffer, Einkommen, Wohnungsbestand, Verkehrsaufkommen errechnet.

4. Die Energieversorgung wird mit Hilfe eines detaillierten EnergiefluBmodelis dargestellt.

5. Der Umweltsektor beschreibt dynamisch die Implementierung von Umweltschutzmaßnahmen.

6. Die Simulation alternativer Strategien oder Szenarien und das Aufzeigen ihrer Auswirkungen erfolgt durch Parametervariation.

Bevor nun exemplarisch einige Ergebnisse von den mit diesem Modell durchgeführten Rechnungen vorgestellt werden, sind noch zwei Anmerkungen erforderlich. Die erste bezieht sich auf die Modellergebnisse, die zweite auf die Modellverifikation.

Es ist nicht das primäre ziel der Modellentwicklung, Prognosen zu liefern, sondern es sollen vielmehr die Folgen alternativer Entscheidungen aufgezeigt und strategien entwickelt werden, mit denen sich energiepolitische Ziele erreichen lassen. Zur Uberprüfung der Modellstruktur und des Modellverhaltens sind zahlreiche Tests durchgefuhrt worden. Dies waren vor allem neben der Analyse des generellen Systemverhaltens uber die Ermittlung der Stoßantwort des Systems auf eine zuvor festgelegte Veränderung wichtiger Parameter, Tests zur Teilverifizierung des Modells an der vergangenen Entwicklung.

In Bild 4 sind für zwei ModellgröBen, den Primärenergieverbrauch und die Primärenergiegewinnung im Inland, der reale und der mit dem Modell errechnete Verlauf für den Zeitraum von 1950 bis 1972 dargestellt. Eine ähnlich gute tbereinstimung zwischen realen und errechneten Werten ergab sich auch bei den anderen wichtigen Modellgrößen, wie z.B. dem Bruttoinlandsprodukt, dem Kapitalstock, den Beschäftigten, den Investitionen usw.

Im folgenden sollen nun einige Ergebnisse, die mit dem vorgestellten Modell erzielt wurden, vorgestelit werden. In Bild 5 ist für den sogenannten Referenz- oder Standardfall die Entwicklung des Bruttoinlandsproduktes und die Veränderung des Wirtschaftswachstums bis zum Jahre 2000 dargestellt. 
Die Ergebnisse dieses Referenzfalles sind in keiner Weise gegenuber den Resultaten anderer Strategieläufe ausgezeichnet. Sie dienen lediglich als Vergleichsbasis bei der Diskussion der Auswirkungen anderer Strategien. Der in diesem Standardfall dargestellten Entwicklung liegen die folgenden Voraussetzungen zugrunde:

Es wird eine möglichst gleichmäßige und stabile Entwicklung innerhalb der gesamten Volkswirtschaft angestrebt. Dazu wird eine kontinuierliche, ungestörte Energieversorgung vorausgesetzt. Unterstellt wird weiter, daB es aufgrund der Produktivitätsbedingungen, trotz einer am Rückgang der Erwerbsquoten und der Bevölkerungszahlen sichtbaren Verringerung des Arbeitskräfteangebotes, durch vermehrten technischen Fortschritt gelingen wird, bis zum Jahre 2000 im Durchschnitt ein stetiges, allerdings abnehmendes Wirtschaftswachstum beizubehalten. Das Bruttoinlandsprodukt würde unter dieser Hypothese von etwa $700 \cdot 10^{9} \mathrm{DM} 1974$ auf ca. $1600 \cdot 10^{9} \mathrm{DM}$ im Jahre 2000 (in Preisen von 62) ansteigen, jedoch mit einer im selben zeitraum von durchschnittlich 4,5 \& auf 2,5 \& verringerten Wachstumsrate.

Bild 6 zeigt den dieser wirtschaftlichen Entwicklung entsprechenden Verbrauch an Primärenergie. Bei der hier zugrundegelegten kontinuierlichen, ungestörten Entwicklung der Energieversorgung wurde unterstellt, $\mathrm{da} B$ aus Gründen der längerfristigen Stabilisierung des Energiemarktes ein relativer Rückgang des Erdolanteils zugunsten anderer Energieträger angestrebt wird, wie es tendenzmäBig der Fortschreibung des Energieprogramms der Bundesregierung entspricht. Dabei steht die forcierte Nutzung der Kernenergie zur Stromerzeugung im Vordergrund. Neue Energietechnologien wie z.B. die Kohlevergasung, die nukleare Fernenergie oder die Nutzung der Wind- und Sonnenenergie sind dabei noch nicht berücksichtigt worden. Der Primärenergieverbrauch steigt unter den für den Standardfall geltenden Prämissen auf fast $800 \cdot 10^{6}$ tSKE im Jahre 2000 an, er wächst also mit 3 \& jährlich. Im Jahre 2000 werden dabei 38 \& des gesamten Primärenergiebedarfs durch Kernenergie gedeckt, während der Verbrauch von Steinbzw. Braunkohle nahezu auf dem heutigen Niveau von $100 \cdot 10^{6} \mathrm{bzw}$. $30 \cdot 10^{6}$ tSKE stagniert und der Anteil des Erdöls von 55 \& auf $30 \%$ zurückgeht. Der Anteil der importierten Fertigprodukte bei Mineralbi dürfte, wenn die derzeitig geltenden Importstrukturen für Mineralöl beibehalten werden, auf rund 40.106 tSKE im Jahre 2000 ansteigen. Der Erdgasanteil hingegen würde sich nach dem Durchlaufen eines Maximums $1985 / 1990$ bis zum Jahre 2000 allmählich wieder verringern, auf rund $80 \cdot 10^{6}$ tSKE, da unterstellt wurde, daB eine nenneswerte VergröBerung des Liefervolumens uber das für die Mitte der 80 er Jahre bekannte MaB hinaus aus Ressourcen-Erschöpfungsgründen nicht möglich sein dürfte. Der Einsatzteil der Wasserkraft und des Importstroms dürfte sich nicht wesentlich verändern.

Die aus einer derartigen Entwicklung des Primärenergieverbrauchs resultierenden Folgen für die Umwelt zeigt Bild 7 anhand der $\mathrm{Zu-}$ bzw. Abnahme der wichtigsten fossilen und radioaktiven Schadstoffemissionen. Aufgrund verstärkter Maßnahmen zur Reduzierung der Umweltbelastung und durch die weniger starke Zunahme des 
Verbrauchs an fossilen Energieträgern, der in den neunziger Jahren sogar absolut rückläufig ist, durchlaufen die mit der Verbrennung fossiler Energieträger verbundenen Schadstoffemissionen in den achtziger Jahren ihren Maximalwert. Die radioaktiven Emissionen nehmen dagegen aufgrund des stark steigenden Einsatzes der Kernenergie bis zum Jahre 2000 stetig zu. Dabei wurde hier mit den den heutigen Erfordernissen der Rückhaltetechnik entsprechenden Emissionsfaktoren für den Reaktor und die Wiederaufarbeitungsanlage gerechnet, d.h. es wurden keine zusätzlichen Rückhaltemaßnahmen eingeführt.

Der mit der im vorangegangenen Bild gezeigten Entwicklung verbundene Strukturwandel in der Energieversorgung führt zu einer Veränderung in der Abhängigkeit von Energieimporten, wie sie in Bild 8 dargestellt ist. Die Importabhängigkeit nimmt bis zum Jahre 2000 stetig $z u$ und erreicht etwa einen Anteil von 808 . Allerdings ist das mit dieser Abhängigkeit verbundene Versorgungsrisiko wegen der unterschiedlichen Versorgungsstruktur im Jahre 2000 ganz anders zu bewerten als im Jahre 1975.

Dj.e Importabhängigkeit von Erdgas und Mineralöl - Energieträgern, die überwiegend aus politisch instabilen Regionen bezogen werden - nimmt bei dem in dieser Rechnung angenommenen Strukturwandel allmählich $a b$, während die Einfuhrabhängigkeit von Kernenergie im gleichen Zeitraum stark zunimmt. Kernbrennstoffe sind jedoch aufgrund ihres hohen Energieinhaltes und der daraus resultierenden günstigeren Reservehaltung und wegen der globaleren Verteilung der Uran- und Thoriumvorräte uber politisch relativ stabile Gebiete vom Standpunkt der Versorgungssicherheit den fossilen Energieträgern weitaus vorzuziehen.

Der positive Effekt dieses Strukturwandels in der Energieversorgung wird auch deutlich, wenn man die für die groBen Energieeinfuhren erforderlichen Einfuhrwerte, denen ein entsprechend grosser DevisenabfluB gegenlibersteht, betrachtet. In Bild 9 ist die Entwicklung der Einfuhrwerte für Erdöl, Mineralölprodukte, Erdgas und Kernenergie für eine bestimmte Preishypothese bis zum Jahre 2000 dargestellt. Natürlich kann über die zukünftige Preisentwicklung keine eindeutige und sicher begründbare Aussage gemacht werden, daher wurde hier nur ein Modellfall von mehreren möglichen betrachtet; bel den für den standardfall geltenden Annahmen würde sich der Devisenaufwand, selbst wenn man optimistischerweise unterstellt, daB die Preise für Erdöl, Mineralölprodukte und Erdgas auf dem Weltmarkt nicht weiter steigen würden,also auf der Preisbasis des Jahres 1974 konstant bleiben wïrden, bis zum Jahre 2000 auf durchschnittlich $40 \cdot 10^{9} \mathrm{DM}$ jährlich belaufen. Für die Kernenergie hingegen würden die Ausgaben unter denselben Prämissen lediglich auf etwa 1.109 DM im Jahre 2000 ansteigen. Allein unter dem Aspekt der Devisenersparnis wäre daher sicher eine noch weiter beschleunigte Einfuhrung der Kernenergie zu begrüBen.

Die Ergebnisse des Standardlaufs zeigen, welche Art von Ergebnissen mit dem Modell zu erzielen sind und welche Fragestellungen zu beantworten sind.

Man kann die Fragenkomplexe in drei Gruppen einteilen: 
1. in Fragen zur wirtschaftlichen Entwicklung und der sich daraus ergebenden Konsequenzen für den Energieverbrauch.

2. in Fragen zur Deckung des Energiebedarfs durch alternative Energieumwandlungs- und -nutzungstechnologien.

3. in Fragen zur Schadstoffbelastung durch den Energieverbrauch.

Aus diesen drei Fragekomplexen sind bereits eine Reihe von Fragestellungen wie z.B. die Auswirkungen eines veränderten wirtschaftlichen Wachstums, die Einführung von EnergieeinsparmaBnahmen, die Einführung neuer Energietechnologien und die Auswirkungen eines verzögerten Kernenergieeinsatzes untersucht worden, deren Ergebnisse hier nicht näher erläutert werden können (siehe dazu $/ 1 /$ ). Neben diesen mehr längerfristigen Fragestellungen sollen nun einige Modellrechnungen erläutert werden, die sich mit der Ermittlung der Folgen von plötzlich auftretenden Versorgungsstörungen befassen. Es handelt sich dabei um die Beantwortung von Fragestellungen der folgenden Art:

- Welche volkswirtschaftlichen Auswirkungen hat eine Störung der Energieversorgung?

- Welche Maßnahmen können im Falle einer Versorgungskrise den wirtschaftlichen Schaden möglichst gering halten?

Bild 10 zeigt beispielhaft die Auswirkungen einer im Jahre 1980 plötzlich auftretenden Lieferkürzung von Mineralöl.

Im einzelnen wurde folgender Ablauf dieses hypothetischen Krisenfalls unterstellt:

Die Einfuhrmengen von Erdöl- und Mineralölprodukten werden $\mathrm{zu}$ Beginn des Jahres 1980 um ein Drittel verringert. Da keine Ersatzlieferungen aus anderen Ländern erfolgen, ist das Mineralölangebot auf dem Inlandsmarkt um ein Drittel kleiner als die Nachfrage. Während einer kurzen tbergangszeit, die durch das Aufbrauchen der Lager bei Verbrauchern, Händlern und Raffinerien gekennzeichnet ist (etwa 100 Tage), werden administrative Verbrauchsbeschränkungen erlassen. Der Mineralölabsatz an private Haushalte, Kleinverbraucher und Industrie wird um rund $40 \%$ reduziert. Die Verkehrsleistung im Individualverkehr wird um $30 \%$, im öffentlichen Personenverkehr um $10 \%$ verringert. Im Güterverkehrsbereich und in der Energiewirtschaft werden keinerlei Beschränkungen einge führt.

Diese administrativen Maßnahmen bedeuten für die privaten Haushalte einen Konsumverzicht, im Produktionssektor kann der Verbrauch aber nur dadurch reduziert werden, daB die Auslastung des Produktionspotentials zurückgenommen wird, d.h. daß die Produktion selbst verringert wird.

In allen Verbrauchssektoren laufen gleichzeitig Prozesse für die Substitution des $81 \mathrm{~s}$ an. Es wird dabei angenommen, daß sich 
die durchschnittliche Substituionszeit infolge der Krisensituation extrem verringert. Die Auswirkungen der Krise verursachen wegen der erforderlichen Einschränkungen einen Produktionsrückgang und führen zu einer starken Rezession des Bruttoinlandsproduktes. Selbst sofort einsetzende Substitutionsmaßnahmen können das wirtschaftliche Wachstum erst ein Jahr später stabilisieren. Das Bruttoinlandsprodukt ist innerhalb eines Jahres von ca. $850 \cdot 10^{9} \mathrm{DM}$ auf $700 \cdot 10^{9} \mathrm{DM}$ zurückgefallen (auch hier wieder in Preisen von 1962 gerechnet); $\in$ s werden weitere viereinhalb Jahre benötigt, um die vor der störung jährlich erbrachte volkswirtschaftliche Leistung wieder zu erreichen.

Der am Bruttoinlandsprodukt gemessene volkswirtschaftliche Verlust würde sich für den Zeitraum von 5 1/2 Jahren auf etwa 825 Mrd. DM belaufen (ermittelt in Preisen von 1962).

Ein anderer wichtiger Punkt im Rahmen der Analyse von Entwicklungsmöglichkeiten der Energiewirtschaft ist die Beschreibung der nachfrageseitig ausgelösten Substitutionsvorgänge zwischen den verschiedenen Endenergieträgern. Die Probleme, die bei der Quantifizierung von Substitutionsprozessen auftreten, liegen darin begründet, daB

$$
\begin{aligned}
& \text { - meist mehr als zwei Energieträger in Konkurrenz stehen, } \\
& \text { daß gerade bei der Auswahl von Energieträgern neben } \\
& \text { den Kosten auch andere Faktoren wie z. B. die Bequem- } \\
& \text { lichkeit und Sauberkeit in der Nutzung der Energie- } \\
& \text { träger eine Rolle spielen, } \\
& \text { und daß Substitutionsvorgänge ausnahmslos dynamischer } \\
& \text { Natur sind. }
\end{aligned}
$$

Die bisher in der konometrie verwendeten Ansätze, die der Beschreibung von Substitutionsprozessen dienen, wie die Kreuzpreiselastitzität, die Substitutionselastizitä oder die exogen vorgegebenen quasi eigengesetzlichen zeitlichen Verläufe der Substitution scheinen für die Darstellung der Substitutionsvorgänge wenig geeignet zu sein. Wir haben deshalb einen Ansatz entwickelt, der versucht, die Substitutionsmechanismen, d.h. die Determinanten und Antriebskräfte der Substitution und ihre Dynamik zu beschreiben. Dieser Ansatz verwendet ein System gekoppelter Differentialgleichungen zur Beschreibung der Substitutionsdynamik und einen Nutzwertansatz zur Erfassung des Einflusses der verschiedenen Bestimmungsgrößen der Substitution.

Die Grundgleichung dinses Ansatzes lautet:

$$
a \frac{d^{3} m_{i}}{d t^{3}}+b \frac{d^{2} m_{i}}{d t^{2}}+\frac{d m_{1}}{d t}+m_{i}=\overline{G N}_{i}(t)
$$

mit $\overline{G N}_{i}(t)=\frac{G_{i}(t)}{\sum_{i} G N_{i}(t)} ; G N_{i}(t)=\sum_{j=1}^{m} g_{j}(t) N_{i, j}(t)$

$$
a=\tau^{3} / 27 ; \quad b=\tau^{2} / 3
$$




$\begin{array}{ll}\mathrm{m}_{i} & =\text { Anteil des Energieträgers } i \text { am Gesamtverbrauch } \\ \tau & =\text { durchschnittliche Substitutionszeit } \\ \mathrm{GN}_{j}(t) & =\text { zeitabhängiger Gesamtnutzen des Energieträgers } i \\ g_{j}(t) & =\text { Gewichtsfaktor des Indikators } j \\ \mathrm{~N}_{i, j}(t) & =\text { Nutzwert des } j \text {-ten Indikators des Energieträgers } i\end{array}$

In diesem Ansatz wird davon ausgegangen, daB sich bei einer Anderung der die Anteile $\mathrm{m}_{i}$ der verschiedenen Energieträger am Gesamtverbrauch bestimmenden Faktoren $\mathrm{GN}_{i}$ die Anteile $\mathrm{m}_{i}$ erst nach einer gewissen Verzögerungszeit, die durch die technischen und ökonomischen Zeitkonstanten einer Umstellung bedingt ist, dem idealen, d.h. stationären Zustand angleichen. Der Gesamtnutzen $\mathrm{GN}_{i}$ eines Energieträgers setzt sich dabei aus gewichteten Teilnutzen für seine Kosten, die Bequemlichkeit seiner Anwendung und seine sonstigen Nutzungseigenschaften zusammen.

Die Anwendbarkeit dieses Ansatzes zur Beschreibung von Substitutionsvorgängen ist an verschiedenen Beispielen der Vergangenheit uberprift worden. Bild 11 zeigt die Ergebnisse einer solchen ex-post-Analyse zur Beschreibung der Substitution für den Raumheizwărmebedarf der privaten Haushalte. Bei diesem Modellbeispiel wurden vier konkurrierende Energieträger (Strom, Gas, Heizöl und feste Brennstoffe) für die Raumheizung betrachtet, wobei jeweils nach Sammel- und Einzelheizungsart unterschieden wurde. Beispielhaft zeigt Bild 11 für den Einsatz der festen Brennstoffe in sammelbeheizten Wohnungen den realen und den modellmäBig errechneten Verlauf, wobei in diesem Fall eine Substitutionszeit von sieben Jahren zugrunde gelegt wurde. Zur Uberprüfung des gewählten Ansatzes sind in das Bild die Ergebnisse einer Sensitivitätsanalyse aufgenommen. Dabei ist einmal der einer subjektiven Bewertung entstammende Gewichtsfaktor des Indikators Bequemlichkeit um 100 \& vergröBert worden, ein anderes Mal ist die durchschnittliche Substitutionszeit um 20 \& erhöht. Diese Resultate und die Ergebnisse anderer Testrechnungen haben gezeigt, daß mit dem gewăhlten Ansatz die Dynamik von Substituionsprozessen relativ zufriedenstellend beschrieben werden kann.

Andere Arbeiten befassen sich mit der Weiterentwicklung des Energieversorgungssektors. Es wurde damit begonnen, den Energieversorgungssektor in ein Modell zur Ermittlung der optimalen Energieversorgungsstruktur bei einem vorgegebenen Endenergiebedarf zu erweitern. Die optimale Struktur der Energieversorgung wird dabei unter Berücksichtigung der jahreszeitlichen Schwankungen des Bedarfs und des Angebots einiger Primärenergieträger ermittelt. Dies scheint besonders wichtig, um zu einer realistischen Beurteilung der Besonderheiten in der Energiewirtschaft z.B. der Möglichkeiten der Wärme-Kraftkopplung oder der Sonnenenergienutzung zu kommen. Denn genau wie bei der Elektrizitătserzeugung ist für die meisten Energieträger die optimale Erzeugungsstruktur auch eine Funktion der Bedarfsschwankungen.

Die Zielfunktion des Optimierungsmodells lautet: 


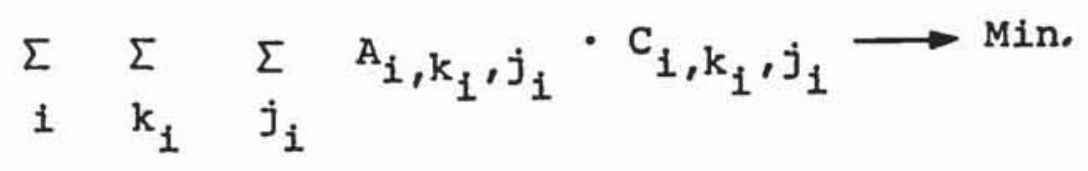

mit $\mathrm{A}=$ spez. Jahreskosten

$i \quad=$ Endenergieträger

$j_{i}=$ Auslastungsbereich

C $\quad$ Kapazität

$k_{i}=$ Technologie

Die spez. Jahreskosten $A_{i, k_{i}, j_{i}}$ der Bereitstellung des Endenergieträgers $i$ mit der Technologie $k_{i}$ und einer Auslastung $j_{i}$ multipliziert mit der entsprechenden installierten Kapazität der Technologie zur Bereitstellung des Endenergieträgers i, aufsummiert über alle Auslastungsbereiche, Energietechnologien und alle Endenergieträger $i$ sollen minimiert werden, oder anders ausgedrückt: Die Kosten für die Bereitstellung aller Endenergieträger sind $z u$ minimieren. Dabei sind eine Reihe von Nebenbedingungen zu beachten, wie z.B.

- Deckung des Bedarfs,

- Kapazitätsbeschränkung oder -vorgabe einer Technologie $\mathrm{k}_{\mathrm{i}}$,

- Koppelproduktionsnebenbedingungen

- Beschränkung oder Vorgabe des Verbrauchs eines Primärenergieträgers,

- $\quad$ und eine Zahl von Umweltrestriktionen.

Mit diesem Ansatz haben wir bereits die Vorteile der wärme-Kraft. Kopplung bei der Deckung des Elektrizitäts- und Fernwärmebedarfs für eine Modellregion untersucht. Anhand dieses Beispiels soll kurz der Anwendungs- und Aussagebereich der Optimierungsrechnung für den Energieversorgungssektor erläutert werden.

Ausgangspunkt war die Frage nach der optimalen Deckung des Elektrizitäts- und Fernwärmebedarfs einer Modellregion mit einem Strombedarf von $10^{9}$ MWe h/a und einem Fernwärmebedarf von $3 \cdot 10^{8}$ MWh/a. Zur Konkurrenz waren insgesamt $8 \mathrm{Kraft-}, \mathrm{bzw}$. Heiz-Kraftbzw. Heizwerke zugelassen, sowohl nukleare als' auch fossile Anlagen. Die Ergebnisse der Rechnungen sind in Tabelle 1 dargestellt. Gegenübergestellt sind hier jeweils die Gesamtkosten zur Erzeugung der Elektrizität und der Fernwärme und der Gesamtwirkungsgrad als ein MaB für die zur Deckung dieses Bedarfs notwendigen Primärenergie.

Im ersten Fall kommt keine Wärme-Kraft-Kopplung zur Anwendung, außerdem geht diese Strategie davon aus, daB keinerlei Kapazitätsbeschränkung (weder nach oben noch nach unten) für die einzelnen betrachteten Umwandlungssysteme bestehen. Es wird also praktisch die optimale zusammensetzung eines Systems, das auf der grünen Wiese errichtet werden soll, ermittelt. Strategie 2 
unterscheidet sich von Strategie 1 nur dadurch, daß hier die Möglichkeiten der Wärme-Kraft-Kopplung genutzt werden. Durch die Einführung der Wärme-Kraft-Kopplung können die Gesamtkosten um etwa 20 \& gesenkt werden, gleichzeitig nimmt aber auch der Wirkungsgrad des Systems um 4,4 of Punkte zu. Diese Zahlen gelten natürlich nur für das hier unterstellte Verhältnis von Elektrizitäts- und Fernwärmeverbrauch von 3:1. Die Strategien 3 und 4 unterscheiden sich von den Strategien 1 und 2 dadurch, daB hier der Realität insofern mehr Rechnung getragen wird, indem für einzelne Umwandlungsverfahren Mindestkapazitäten oder für einzelne Primärenergieträger (z.B. Kohle) Mindestverbräuche vorgeschrieben werden. Aber auch diese Restriktionen, die in unserem Fall zu Lasten der Wärme-Kraftkopplung gehen, zeigen die deutlichen Vorteile der Wärme-Kraftkopplung. 10 \% geringere Kosten führen gleichzeitig zu einem Anstieg des Wirkungsgrades um 3 \& Punkte.

Mit diesen Ausführungen ist der derzeitige Stand der Modellentwicklung in unserer Arbeitsgruppe gekennzeichnet. Zum AbschluB dieser Präsentation scheint es jedoch angebracht, noch einmal auf die Anmerkungen, die eingangs gemacht wurden, zurückzukommen:

Ein Modell ist immer nur ein vereinfachter Ausschnitt der Wirklichkeit, der so gewählt wurde, daß die Simplifizierung im Rahmen der Zielsetzungen zulässig sind. Es gibt deshalb kein "Supermodell", das Antworten auf alle Fragen ermöglicht. Die Entwicklung von Energiemodellen steht heute erst am Anfang, aber uns scheint es sicher, daB die Aussagemöglichkeiten, die Aussagequalitäten und damit der Nutzen der Modellentwicklung mit zunehmender Erfahrung grösser werden.

\section{Literaturhinweise}

/1/ St. RATH-NAGEL:

Alternative Entwicklungsmöglichkeiten der Energiewirtschaft in der BRD - Untersuchung mit Hilfe eines Simulationsmodells, Dissertation RWTH Aachen in Vorbereitung.

12/ A. voss:

Ansătze zur Gesamtanalyse des Systems Mensch - Energie - Umwelt. JlL-982-RG, Jul1 1973.

/3/ F. NIEHAUS:

Langzeitaspekte der Umweltbelastung durch Energieerzeugung: $\mathrm{CO}_{2}$ und $\mathrm{B}^{3}$. JUL-1165-SE. 


\section{Allgemeines Ziel}

Analyse der Entwicklungsmöglichkeiten des Energiesystems der BRD

\section{Teilziele}

Struktur eigenschaften dynamisches Verhalten

Kritische Faktoren

Grenzsituationen

Folgen alternativer

Strategien

Eingriffs - und

Steuerungsmöglichkeiten

Bild 1: Zielsetzungen der Modellentwicklungen 


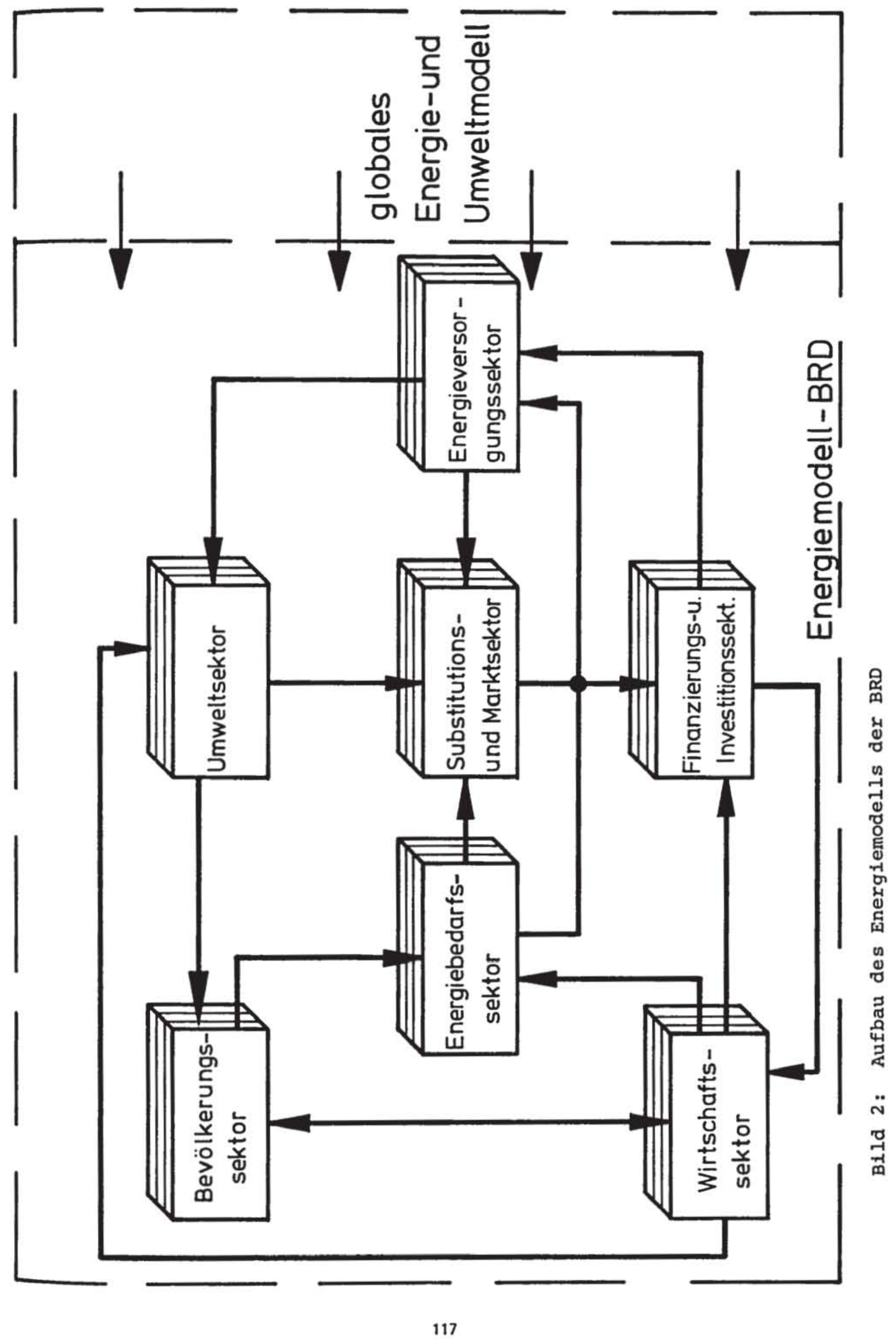




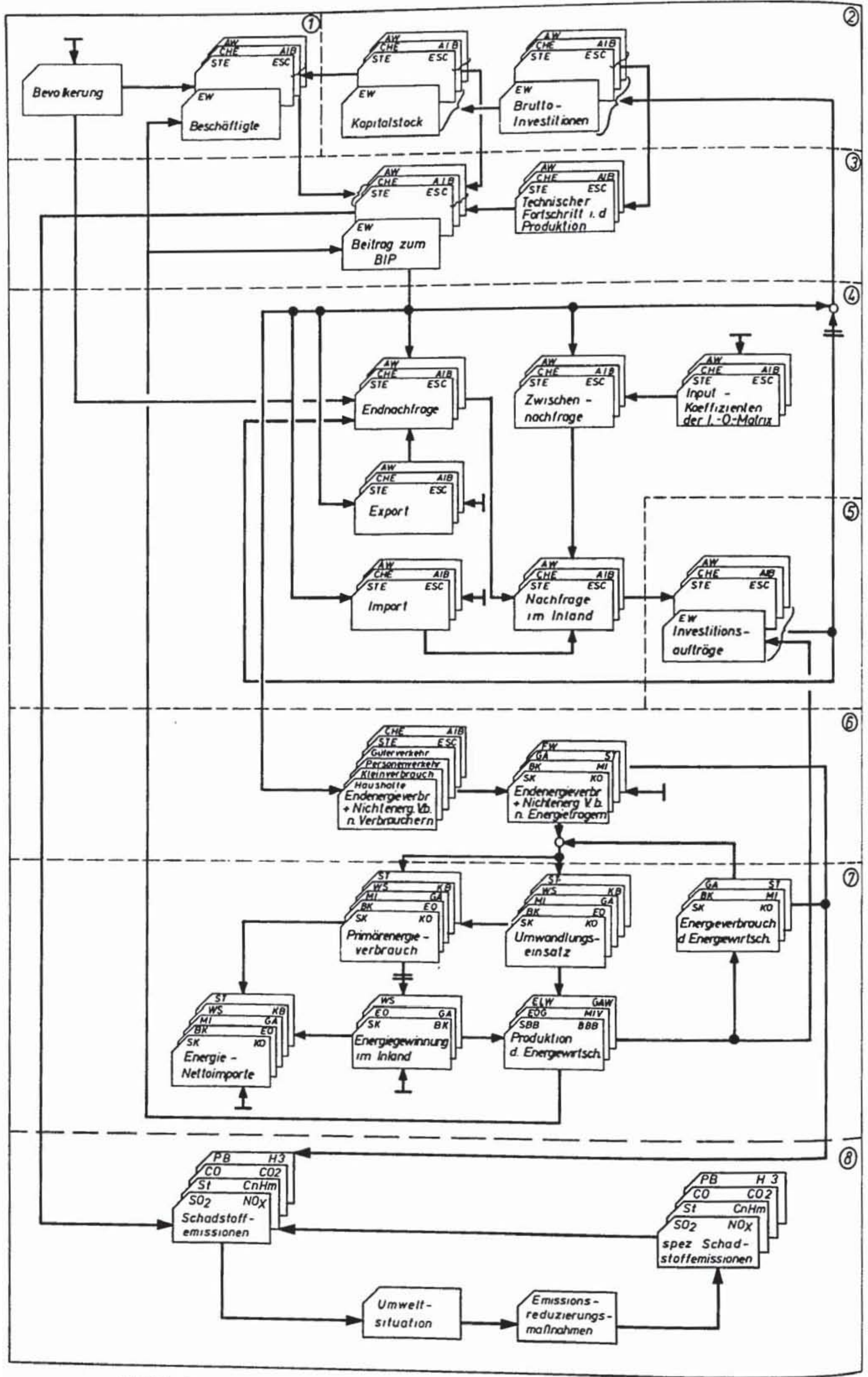

Bild 3: Grundstruktur des Energiemodells der BRD 
Erläuterungen zur Modellstruktur (Bild 3):

Modellsektoren:

1. Bevölkerungs- und Beschäftigungssektor

2 Kapitalsektor

3 Produktionssektor Wirtschaftssektor

4 Nachfragesektor

5 Investitionssektor

6 Energieverbrauchs-

sektor

7 Energieversorgungs-

sektor

8 Umweltsektor

Wirtschaftsgruppen EW Energiewirtschaft

SBB Steinkohlenbergbau

BBB Braunkohlenbergbau

EOG Erdöl- und Erdgasgewinnung

MIV Mineralölverarbeitung

ELW Elektrizitätswirtschaft

GAW Gaswirtschaft

STE Industrie der Steine und Erden

ESC Eisenschaffende Industrie

CHE Chemische Industrie

AIB Andere industrielle Bereiche

AW Andere Wirtschaftsbereiche

Energieträger:

FB Feste Brennstoffe

SK Steinkohle

KO Koks

EO Erdöl

MI Mineralölprodukte

HO Heizöl

FK Flugkraftstoffe

VK Vergaserkraftstoffe

DK Dieselkraftstoffe

GA Gas

WS Wasserkraft

KB Kernbrennstoffe

ST Strom

FW Fernwärme

Schadstoffe:

$\begin{array}{cl}\mathrm{SO}_{2} & \text { Schwefeldioxyd } \\ \mathrm{NO}_{\mathrm{x}} & \text { Stickoxyde } \\ \mathrm{ST} & \text { Stäube } \\ \mathrm{C}_{\mathrm{n}}{ }_{\mathrm{H}} & \text { Kohlenwasserstoffe } \\ \mathrm{CO} & \text { Kohlenmonoxyd } \\ \mathrm{CO}_{2} & \text { Kohlendioxyd } \\ \mathrm{PB}_{\mathrm{H}} & \text { Blei } \\ \mathrm{H}-3 & \text { Tritium }\end{array}$




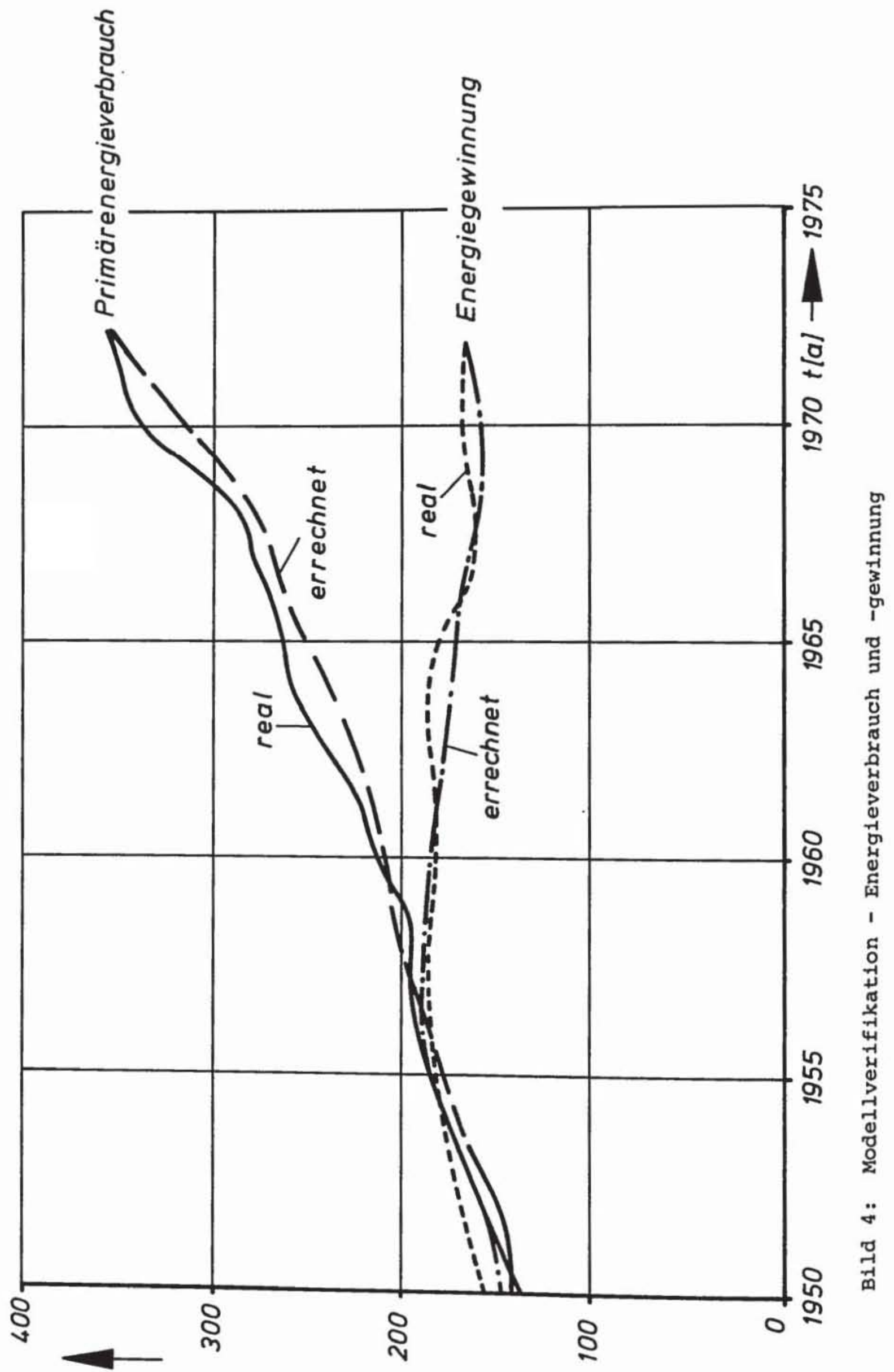

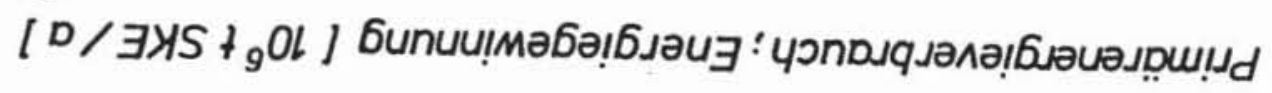




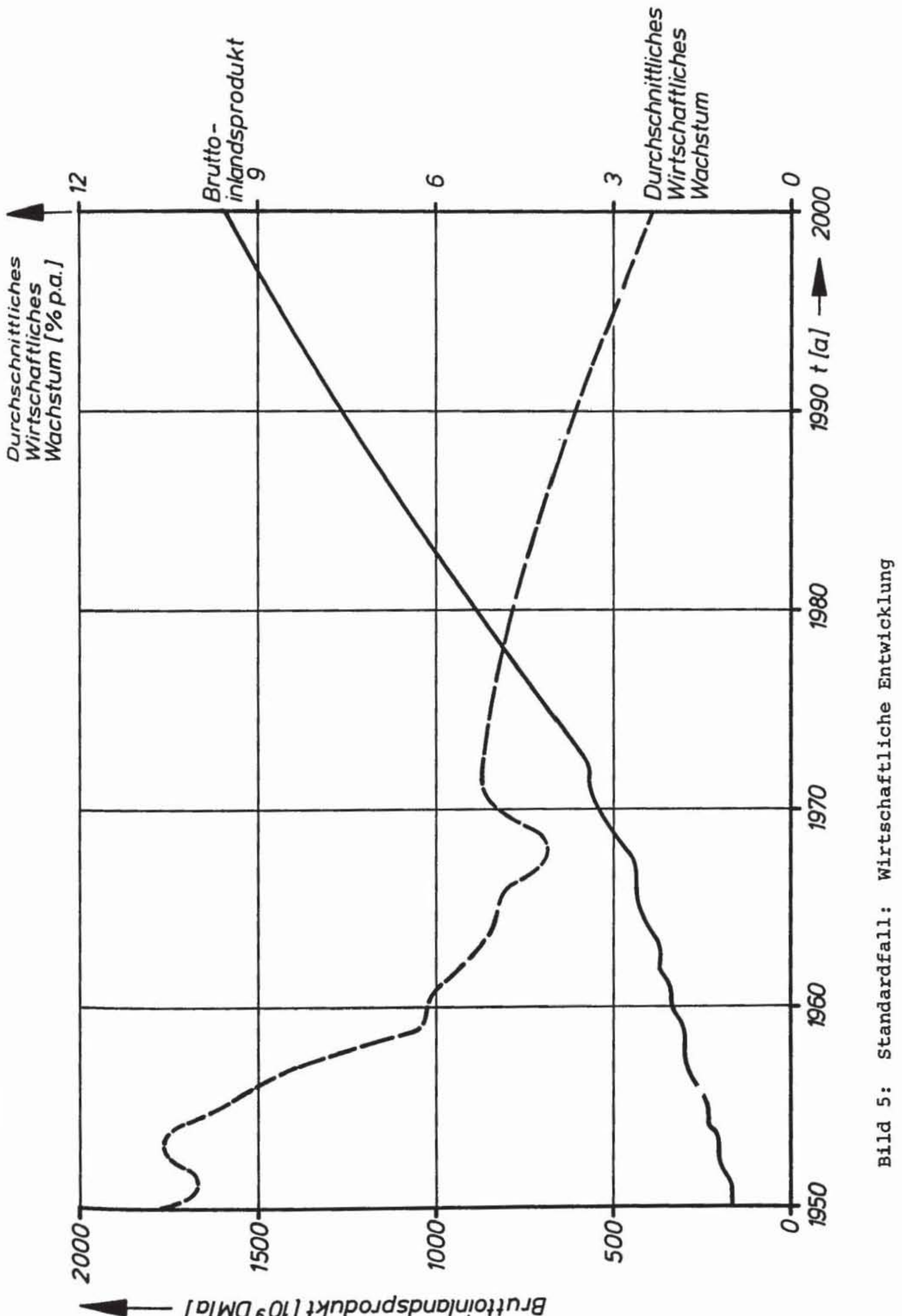




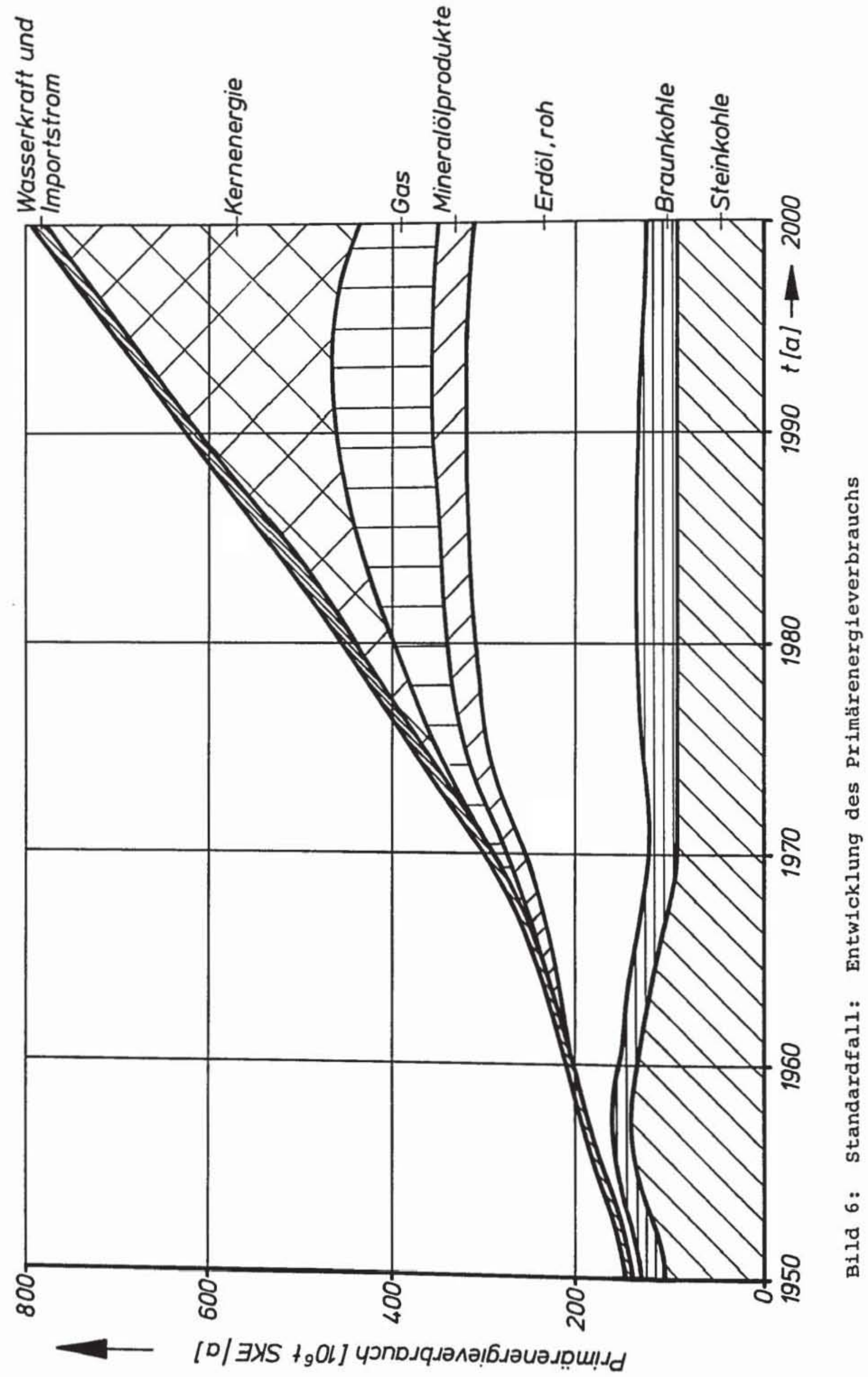




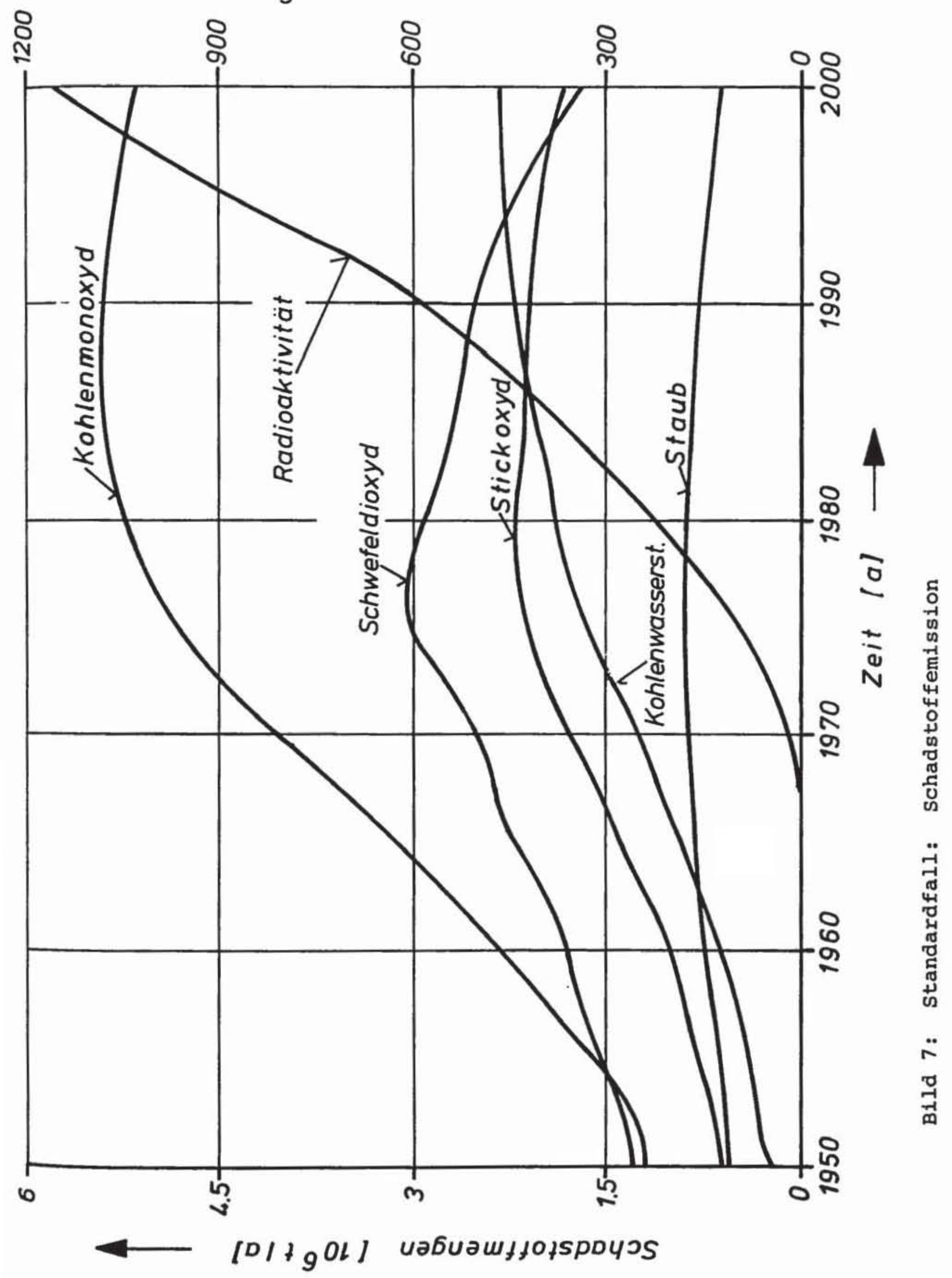




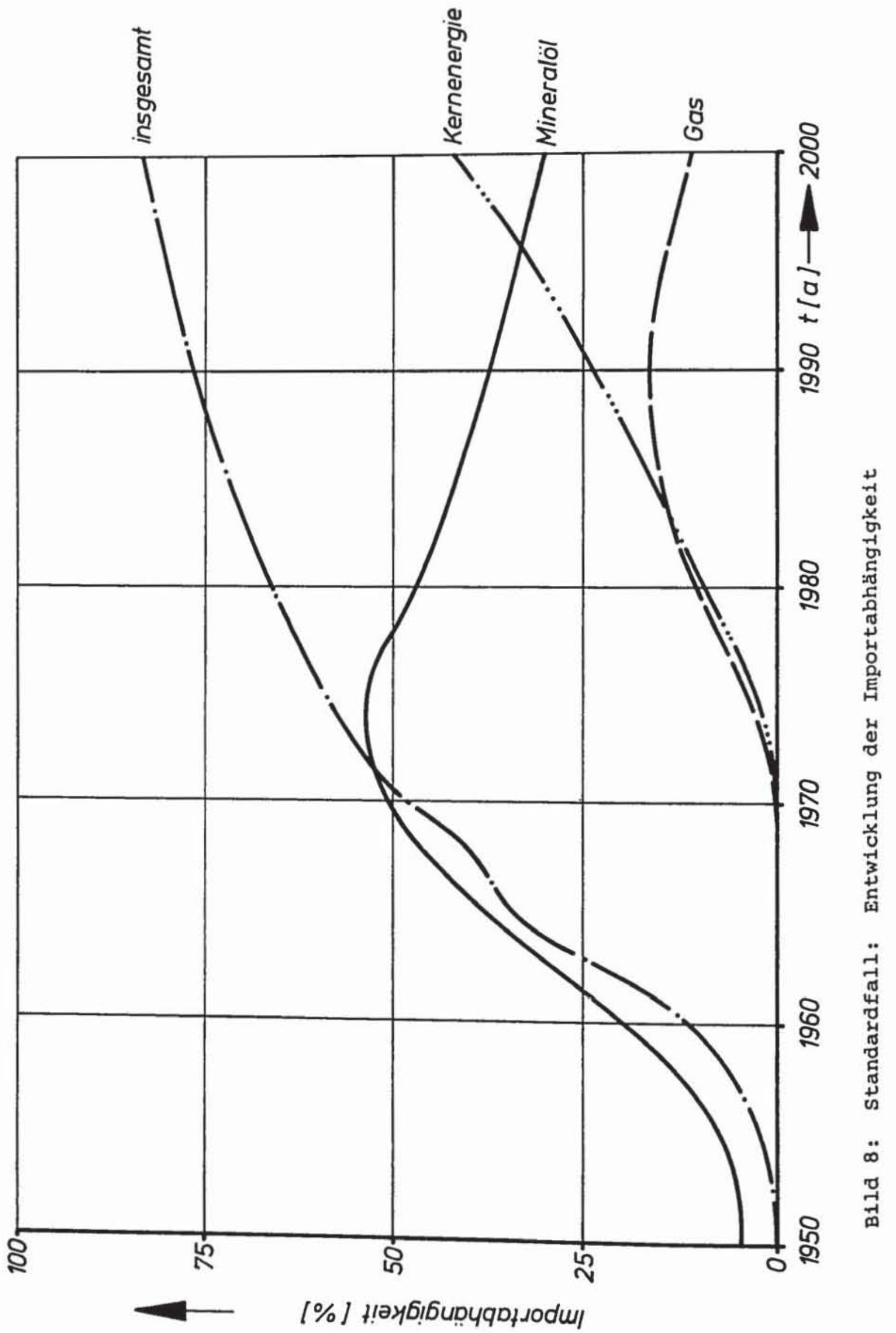




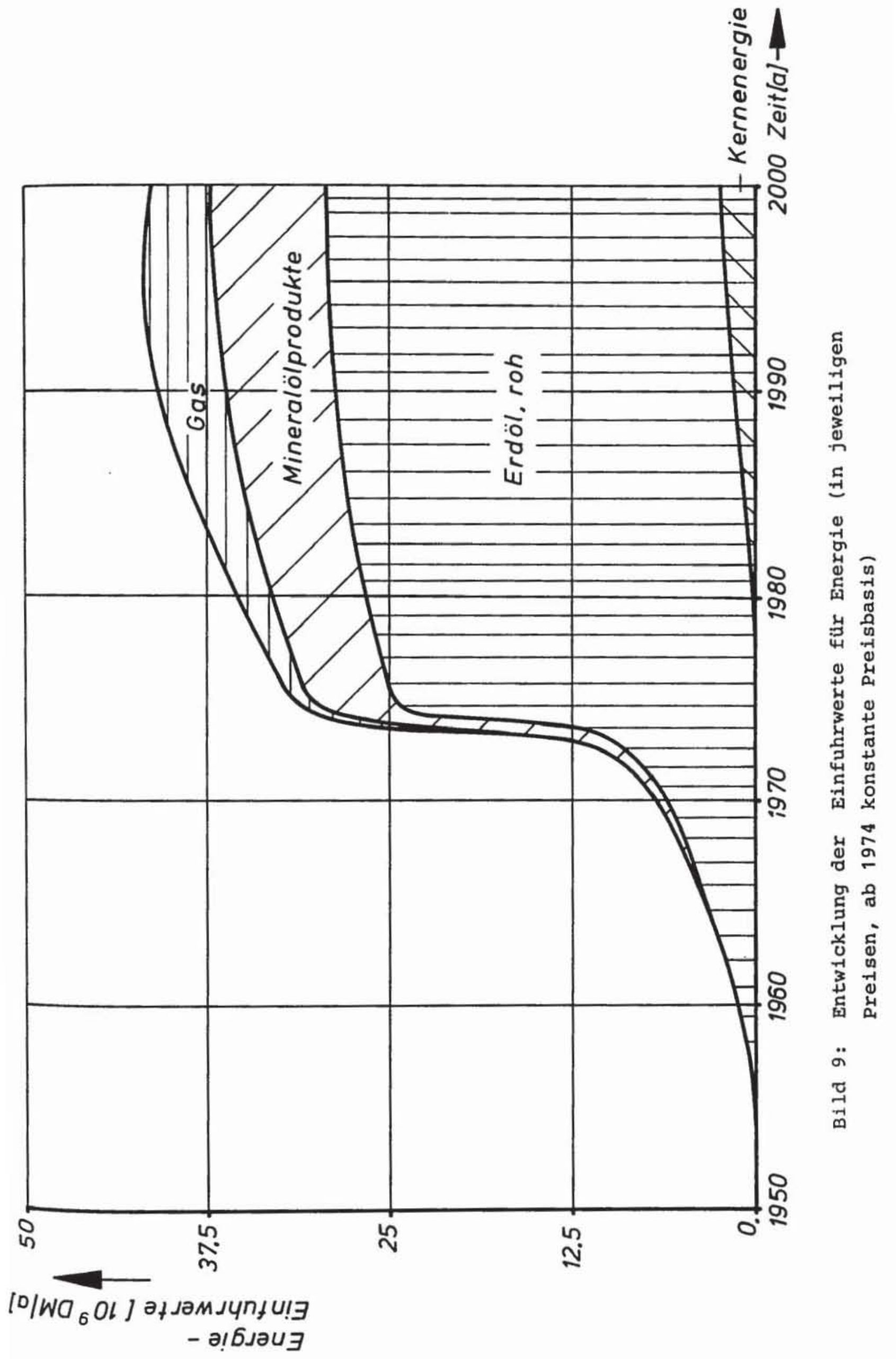




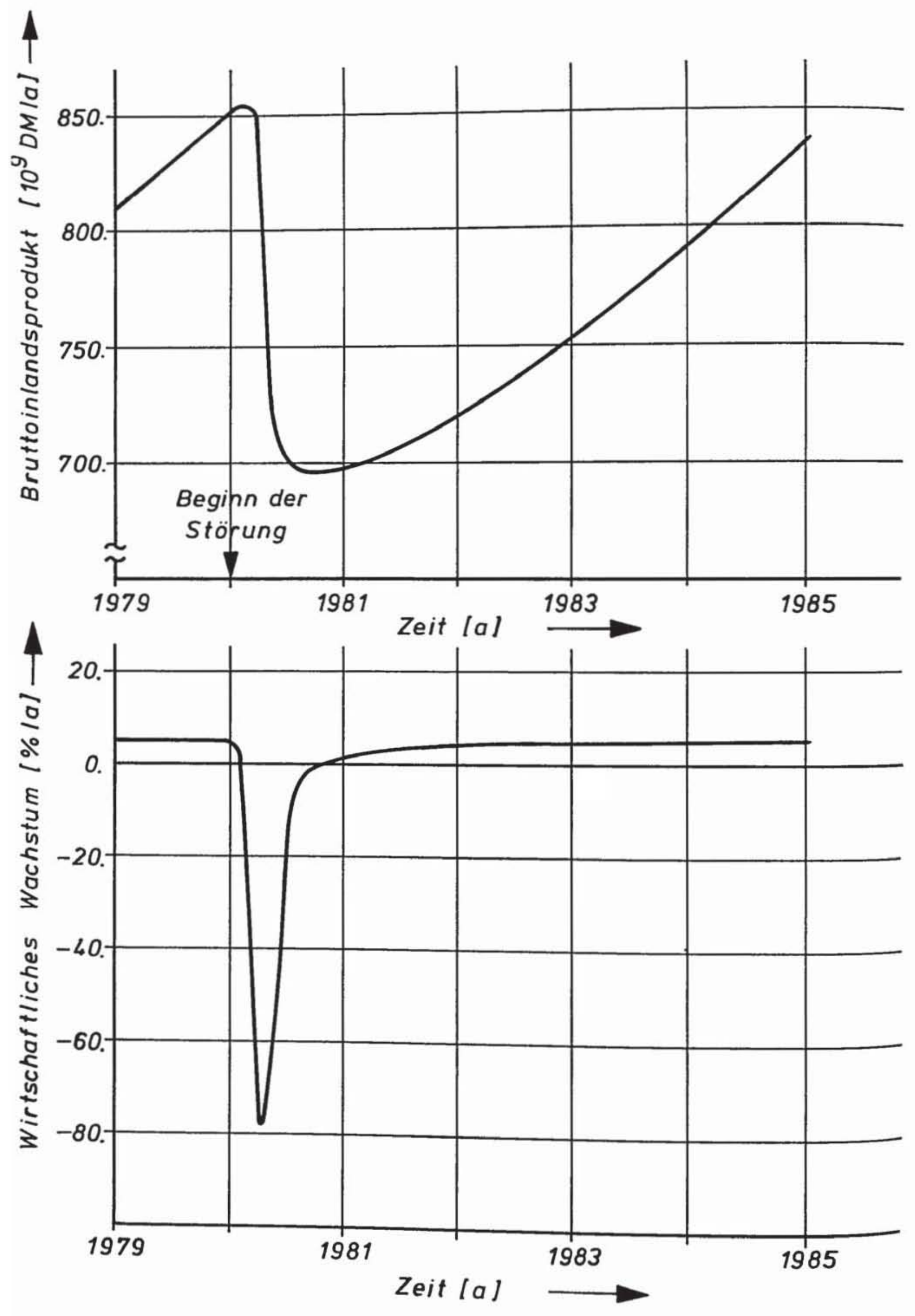

Bild 10: Versorgungskrise 
— realer Verlauf

- - errechneter Verlauf

- - Substitutionszeit $20 \%$ erhöht

- Gewichtsfaktor um 100\% vergrößert

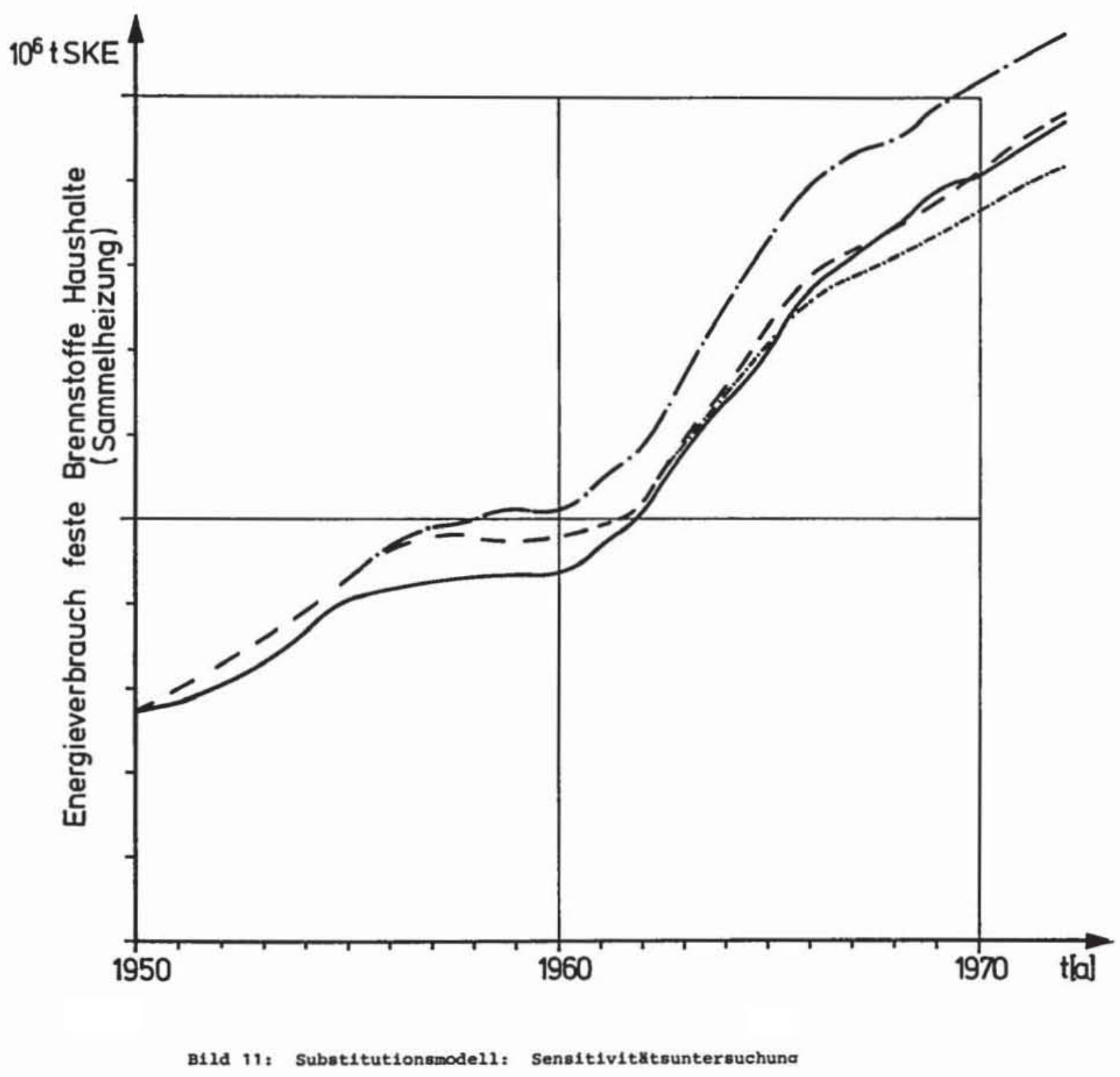




\begin{tabular}{|c|l|c|c|c|}
\hline Strategie & $\begin{array}{l}\text { Wärme-Kraft } \\
\text { Kopplung }\end{array}$ & $\begin{array}{c}\text { Verbrauchs- } \\
\text { restriktionen }\end{array}$ & $\begin{array}{c}\text { Gesamtkosten } \\
{\left[10^{9} \mathrm{DM} / \mathrm{a}\right]}\end{array}$ & $\begin{array}{c}\text { Gesamtwirkungs- } \\
\text { grad [\%] }\end{array}$ \\
\hline 1 & & & 48,4 & 42,8 \\
\hline 2 & $x$ & & 40,7 & 47,2 \\
\hline 3 & & $x$ & 49,3 & 37,9 \\
\hline 4 & $x$ & $x$ & 45,0 & 40,9 \\
\hline
\end{tabular}

Tabelle 1: Optimierungsergebnisse für eine Modellregion 